\title{
Time Trend and Premature Death Due to Diabetes Mortality in a Disadvantage Mountainous Province of Viet Nam From 2005 To 2018
}

\author{
Long Cong Nguyen ${ }^{1 \#}$, Nga Thu Hoang ${ }^{2 \#}$, Nghia Van Luu ${ }^{3}$ and Ngoan Tran Le ${ }^{4,5 *}$ \\ ${ }^{1}$ Gastroenterology \& Hepatology Center, Bach Mai Hospital, Hanoi, Viet Nam \\ ${ }^{2}$ The Department of Endocrinology, Hanoi Medical University Hospital, Hanoi city, Viet Nam \\ ${ }^{3}$ Lang Son Provincial Center for Diseases Control (CDC), Lang Son city, Viet Nam \\ ${ }^{4}$ Institute of Research and Development, Duy Tan University, Da Nang, Viet Nam \\ ${ }^{5}$ Department of Public Health, School of Medicine, International University of Health and Welfare, Japan \\ \#These Authors shared the lead authors
}

*Corresponding author: Ngoan Tran LE, Department of Public Health, School of Medicine, International University of Health and Welfare, Japan

ORCID:

Ngoan Tran Le: 0000-0001-7862-492X

Long Cong Nguyen: 0000-0002-5275-9014

\section{ARTICLE INFO}

Received: 嶓 October 21, 2021

Published: 㹃 November 11, 2021

Citation: Long Cong Nguyen, Nga Thu Hoang, Nghia Van Luu, Ngoan Tran Le. Time Trend and Premature Death Due to Diabetes Mortality in a Disadvantage Mountainous Province of Viet Nam From 2005 To 2018. Biomed J Sci \& Tech Res 39(5)-2021. BJSTR. MS.ID.006374.

Abbreviations: DM: Diabetes Mellitus; IDF: International Diabetes Federation; WHO: World Health Organization

\section{ABSTRACT}

Objectives: This study determined time trend and proportion of death under the age of 70 years due to Diabetes mellitus (DM) at the mountainous Lang Son province.

Methods: We accessed a database of population-based mortality registration of the Lang Son province to derive 426 MD cases during 2005-2018. The variables of each case included patient ID, age, sex, date, and the underlying cause of death. Agestandardized rate per 100,000 person-years using the WHO standard population (ASR) and the adjusted mortality rate ratio and 95\% confidence interval (MRR, 95\%CI) were estimated.

Findings: When combined all cases from 2005-2018, the ASR rate per 100,000 person-years was 5.8 in both genders, 9.4 in men and 2.7 in women. The mortality rate increased significantly over the period for men but not in women, with a per-year increment mortality rate ratio MRR $(95 \% \mathrm{CI}): 1.041(1.014,1.069), \mathrm{p}=0.003$, and MRR (95\%CI): $1.011(0.967,1.057), p=0.619$, respectively. A proportion of premature deaths under the age of 70 years was about three-fourth.

Conclusions: The findings warrant further studies and support to vulnerable people in remote areas to prevent premature mortality due to this preventable disease.

\section{Introduction}

Diabetes Mellitus (DM) is a chronic disease with multiple complications that contribute to serious illnesses such as cardiovascular disease, kidney disease, eye disease, nerve damage, and lower limb amputation. Reports from the International Diabetes Federation (IDF) in 2019 show that approximately 463 million people are living with DM, of which $79 \%$ of people live in low and middle-income countries [1]. The prevalence of DM tends to increase continuously in the future and is estimated to reach $7.7 \%$ and 439 million adults by 2030 [2]. Approximately $75 \%$ of people with DM and $81 \%$ of people with DM who are undiagnosed live in low and middle-income countries [3]. In addition to serious complications, people with DM also have a higher risk of morbidity and mortality than the general population. As reported from IDF 
in 2015, it was estimated that five million deaths were caused due to DM [4]. According to the World Health Organization (WHO), DM was the seventh leading cause of death and directly caused an estimated 1.6 million deaths in 2016 [5]. Consistent with the global trend, in Viet Nam, the prevalence of DM has estimated to be nearly doubled over the past 10 years [6].

A national survey in 2012 showed that the prevalence of DM and prediabetes were $5.4 \%$ and $13.7 \%$, respectively [7]. Another study of Ngoc Minh Pham in Thai Nguyen province which was conducted from 2011 to 2013 reported standardized prevalence rates of diabetes and prediabetes were $6.0 \%$ and $13.5 \%$, respectively [8]. Pham also estimated that by 2035 , these figures will respectively be $7.0 \%$ and $15.7 \%$. It was estimated that in 2015 , about 3.5 million adults aged 20-79 years had DM, and it was responsible for 53,400 deaths [6]. These findings suggest an increasing trend in the diabetic population in Viet Nam and the burden for medical care of the country. However, the data on population-based mortality due to DM has not been available to date in Viet Nam. Mortality is an important measure to assess the burden of DM and also a vital aspect in interventions and treatment of DM. To the best of our knowledge, data of diabetes from a population-based mortality registration is limited, suggesting further studies for more accurate estimates in Viet Nam. Additionally, there is a rapid increase in diabetes prevalence in rural populations worldwide in recent years [9].

Nonetheless, even though two-thirds of the population live in rural areas [10], researches on this issue in Viet Nam is still lacking. Lang Son is a mountainous province in the northeastern part of Viet Nam, with a population of over 768,700 in 2016, of which nearly $80 \%$ live in rural areas. There are seven major ethnic groups, with only $16.5 \%$ being Kinh and the remainder designated ethnic minorities [11]. Ethnic minorities living in rural and mountainous areas often face difficulties in accessing health care services as well as paying for diabetes treatment, which enhances the risk of mortality due to diabetes complications. This study determined time trend and proportion of death under the age of 70 years due to DM at the mountainous Lang Son province.

\section{Materials and Methods}

We accessed a database of population-based mortality registration of the Lang Son province to derive 426 MD cases (ICD-10: E10-E14, 317 men and 109 women) during 2005-2018. Variables of each case included patient ID, age, sex, date, and the underlying cause of death with ICD-10 coding for each year, and the yearly average population by sex and age[12,13].

\section{Study Population and Setting}

Lang Son is located in the Northeast of Viet Nam, far about $154 \mathrm{~km}$ from the Hanoi capital. While the Northern-Eastern region borders to China, the rest of Lang Son province borders to five other provinces belonging to Viet Nam, including Cao Bang, Bac Can, Thai Nguyen, Bac Giang, and Quang Ninh [14], (Figure 1). The primary purpose of the population-based mortality registration was conducted in the whole country at 64 provinces/cities to count cancer mortality during 2005-2006 in Viet Nam including Lang Son province $[15,16]$, thereafter, the work was done every year and we conducted a validation study on the quality of data of mortality registration of the completeness and accuracy due to injury and all causes [17,18]. From 2005 to 2018, the mega-database of 49,253 deaths due to all causes including 426 DM deaths with case ID, age, sex, date, and the underlying cause of death with ICD-10 coding for each year was created for further research and study.

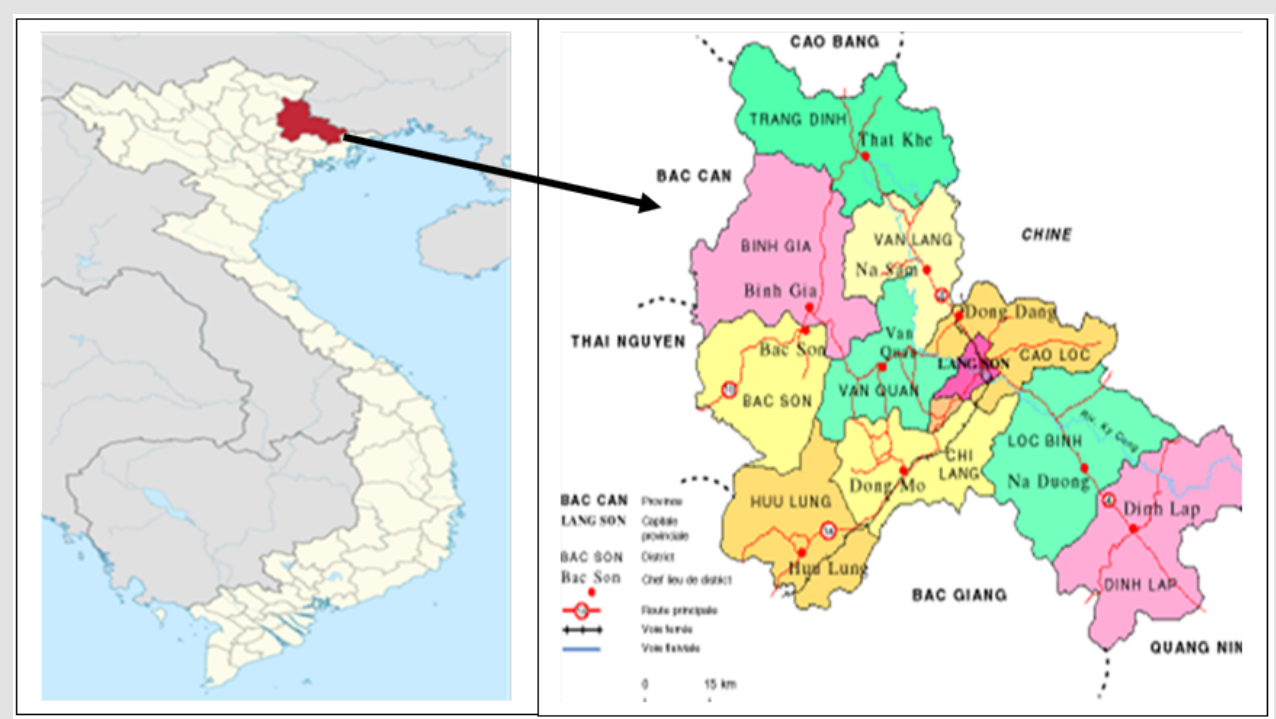

Figure 1: Location and map of the Lang Son province. 


\section{Healthcare Systems and Facilities}

Healthcare systems and facilities in 2016 of the Lang Son province [14] had four provincial hospitals having 810 beds, 10 district hospitals having 1,045 beds, 25 regional clinics having 145 beds, and 226 commune health stations having 678 beds in providing healthcare services at all regions of the province. The total number of Medical Doctors was 680, giving the number of physicians per 10,000 inhabitants was 8.8. People aged forty or older can visit these health facilities to test for DM and receive treatment if they are suffering from DM.

\section{Validation of Mortality Registration in Vietnam and Lang Son Province}

Quality of mortality registration for all causes including DM was examined for completeness and accuracy in Viet Nam and the mortality registration systems at the Lang Son province have been operating these systems following the same regulation guideline by the Ministry of Health [18]. The estimated completeness was $93.7 \%$ in 2008 at three provinces represented three regions of Viet Nam (North, Central, and South) [19]; was 81\% at 16 provinces in 2009 represented by sixty-three provinces/cities nationwide [20]. For data of DM, a pilot study in another province of Nghe An in 2014, the estimated sensitivity and specificity were 50\%-57\% and 99\%$100 \%$, respectively [21]. In Quang Ninh and Thai Nguyen provinces, [22] bordering with the Lang Son province (Figure 1), the estimated Kappa for DM was $33 \%$.

\section{Inclusion and Exclusion of the Study Subjects}

Data on the status and distribution of mortality recorded in Lang Son province during 2005-2018 (missing 2009 and 2010) was calculated using the following input progress:

a) A list of deaths due to all causes according to the "Report card of the cause of death" in all 226 State Commune Health Stations of eleven cities/districts in Lang Son province was checked, including 5 variables: Patient ID, age, gender, date, month, year of death and cause of death (according to ICD-10).

b) All deaths according to the criteria of deaths due to DM (ICD10: E10-E14) were chosen.

c) All confirmed deaths not due to DM (ICD-10: E10-E14) were excluded from the present study.

\section{Data Analysis}

Data collected were entered and analyzed by EXCEL and STATA 10.0 software. The list of deaths due to diabetes is presented by gender, age group, district, and in general for the whole province. The death rate per 100,000 people by gender, age group, district, and the whole province was calculated. Age-standardized rate per 100,000 person-years (ASR) using the WHO standard population (2000-2025) and the adjusted mortality rate ratio and $95 \%$ confidence interval (MRR, 95\%CI) were estimated by sex and year.

\section{Ethical Approval}

Investigation of the cause of death does not interfere with the body of the person providing the data and does not harm the health of the study participants. The information on the cause of death will be kept confidential when presenting does not show the name and address of the person who died. The Hanoi Medical University Review Board in Bio-Medical Research \# 61/HMURB, dated 25 November 2008, and the Board of Ethics in Bio-Medical Research at University of Medicine and Pharmacy at Ho Chi Minh City \#106/ UMP-BOARD, dated 20 March 2019 have approved the present research protocol.

\section{Results}

Among 49,253 death cases for 9,077,500 person-years, the total number of deaths due to DM was 426 cases, which was estimated for $0.86 \%$. The proportion of deaths aged under 70 years old was highest in 2014 , at $87.5 \%$ out of total death cases due to DM occurrence in this year and about three-fourth of total deaths in 2005-2018. Diabetic mortality was lowest in 2005, at 22 deaths; the number of death was highest in 2018, at 48 cases. Consequently, the mortality crude rate (per 100,000 person-years) was lowest in 2005, at 3.1, and highest in 2018, at 6.1. Using the WHO standard population, the age-standardized mortality rate (WHO-ASR) was still highest in 2018, at 8.0; when combined all cases from 20052018 and in both genders, this rate was 5.8 per 100,000 personyears. Per-year increment MRR (95\%CI) was 1.033 (1.010, 1.057) and this result was statistical significance $(p=0.005)$, (Table 1). Among 31,262 total cases, there were 317 deaths due to DM in men, which was estimated for $1.01 \%$. The proportion of deaths aged under 70 years old was highest in 2014, at $96.3 \%$. Diabetic mortality was lowest in 2005, at 15 deaths; the number of death was highest in 2016, at 38 cases. As a result, the mortality crude rate (per 100,000 person-years) was lowest in 2005, at 4.2, and highest in 2016, at 9.7.

According to the WHO standard population, WHO-ASR was highest in 2018, at 14.3; when combined all cases from 20052018 and in men, this rate was 9.4 per 100,000 person-years. Peryear increment MRR $(95 \% \mathrm{CI})$ was $1.041(1.014,1.069)$, and this result was statistical significance $(\mathrm{p}=0.003)$, (Table 2$)$. In general, the number of deaths due to DM in women was lower than that in men. Among 17,990 total deaths, 109 deaths were due to DM in women, which accounted for $0.61 \%$. The proportion of deaths 
aged under 70 years old was highest in 2007, at $72.7 \%$. Diabetic mortality was lowest in 2017, at 4 deaths; the number of death was highest in 2015, at 16 cases. The mortality crude rate (per 100,000 person-years) was lowest in 2017, at only one and highest in 2015, at 4. Using the WHO standard population, WHO-ASR was highest in 2015, at 4.7; when combined all cases from 2005-2018 and in women, this rate was 2.7 per 100,000 person-years. Peryear increment MRR $(95 \% \mathrm{CI})$ was $1.011(0.967,1.057)$, but this result was not statistically significant $(\mathrm{p}=0.619)$, (Table 3). Figure
2 shows the difference in age-specific mortality rates per 100,000 person-years between men and women during the survey period. In general, the mortality rate in men was much higher in women. Also, the number of deaths due to DM consistently higher with increasing age. In 0-29 age groups, the proportion of deaths due to DM was very low (lower than 1\%) in both genders. The mortality rate of men, women, and in both genders was highest in people who were 80 years old and older, at 53.3, 38.2, and 42.8, consequently.

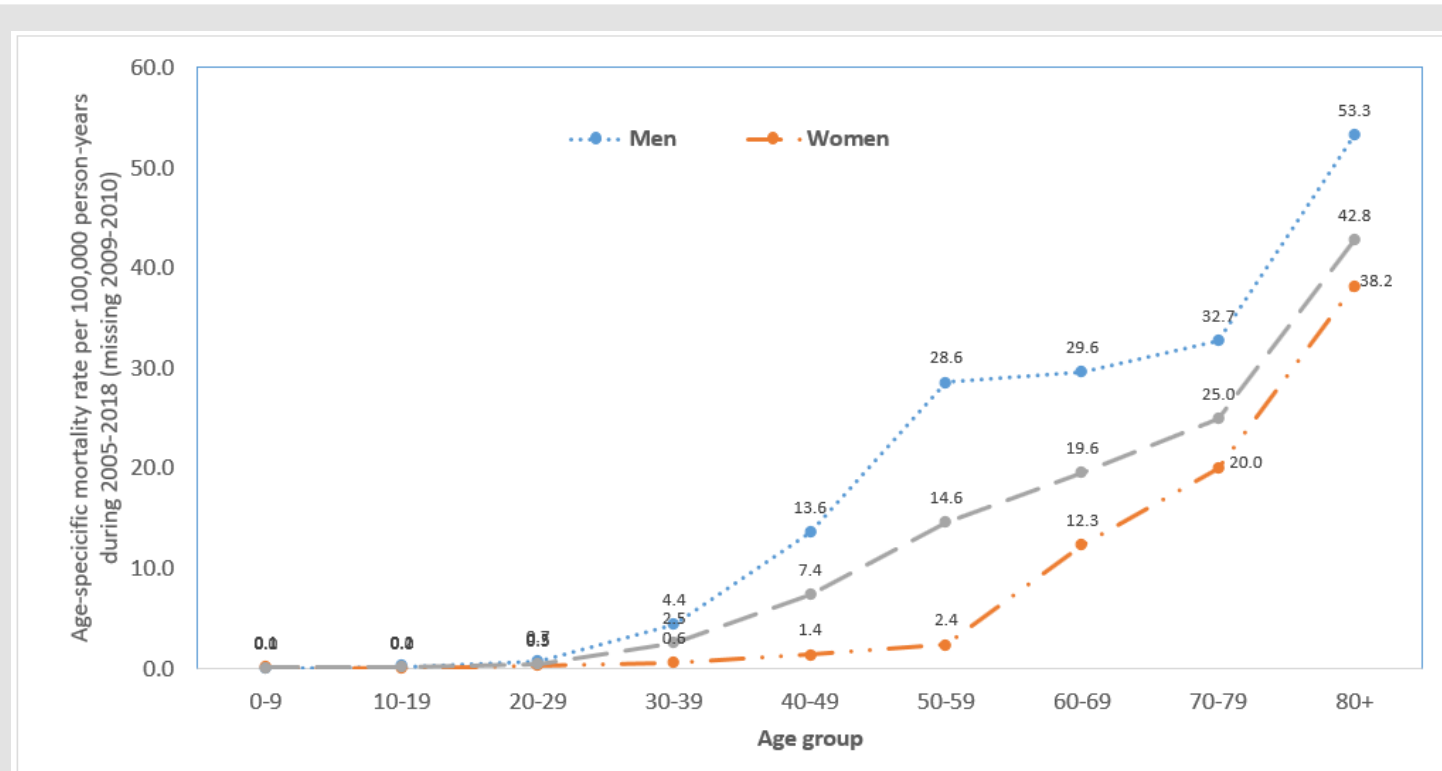

Missing data for the year of 2009-2010;

Figure 2: Age-specific mortality rate per 100,000 person-years by sex during 2005-2018.

Table 1: Mortality due diabetes in both genders from 2005 to 2018 in Lang Son province.

\begin{tabular}{|l|c|c|c|c|c|c|}
\hline Year & Case & Crude rate \$ & \%<70 \# & WHO-ASR \$ & MRR (95\%CI) \$\$ & p \\
\hline 2005 & 22 & 3.1 & 63.6 & 3.5 & 1 (reference) & 0.431 \\
\hline 2006 & 28 & 3.8 & 75.0 & 4.5 & $1.251(0.716,2.187)$ & 0.131 \\
\hline 2007 & 34 & 4.6 & 73.5 & 5.8 & $1.512(0.885,2.586)$ & 0.471 \\
\hline 2008 & 28 & 3.8 & 78.6 & 4.5 & $1.228(0.703,2.147)$ & 0.080 \\
\hline 2011 & 36 & 4.9 & 80.6 & 6.1 & $1.606(0.945,2.730)$ & 0.116 \\
\hline 2012 & 35 & 4.7 & 71.4 & 5.7 & $1.533(0.899,2.613)$ & 0.106 \\
\hline 2013 & 35 & 4.7 & 71.4 & 5.7 & $1.552(0.910,2.645)$ & 0.052 \\
\hline 2014 & 40 & 5.1 & 87.5 & 6.1 & $1.675(0.995,2.818)$ & 0.027 \\
\hline 2015 & 43 & 5.5 & 74.4 & 6.7 & $1.787(1.069,2.987)$ & 0.009 \\
\hline 2016 & 47 & 6.0 & 78.7 & 7.5 & $1.959(1.180,3.250)$ & 0.454 \\
\hline 2017 & 30 & 3.8 & 73.3 & 4.7 & $1.234(0.712,2.139)$ & 0.007 \\
\hline 2018 & 48 & 6.1 & 68.8 & 8.0 & $2.003(1.209,3.317)$ & \\
\hline
\end{tabular}

Note: Missing data for the year of 2009-2010; the estimated proportion of deaths due to diabetes was $0.86 \%$ (426 cases of diabetes vs. 49,253 total cases), both genders. \$ adjusted for age group (0-9, 10-19, 20-29, 30-39, 40-49, 50-59, 60-69, 70-79, 80+) and sex. Per-year increment MRR (95\%CI): 1.033 (1.010, 1.057), p=0.005. \$ Crude rate per 100,000 person-years; \$ Age-standardized rate per 100,000 person-years using the World Health Organization standard population for 2000-2025; \# Proportion of death cases aged under 70 year-olds. When combined all cases from 2005-2018, both genders, WHO-ASR: 5.8 per 100,000 person-years. 
Table 2: Mortality due diabetes in men from 2005 to 2018 in Lang Son province.

\begin{tabular}{|c|c|c|c|c|c|c|}
\hline Year & Case & Crude rate \$ & $\mathbf{9}<\mathbf{7 0 ~ \#}$ & WHO-ASR \$ & MRR (95\%CI) \#\# & p \\
\hline 2005 & 15 & 4.2 & 66.7 & 5.7 & 1 (reference) & 0.525 \\
\hline 2006 & 19 & 5.2 & 84.2 & 6.2 & $1.245(0.633,2.451)$ & 0.222 \\
\hline 2007 & 23 & 6.3 & 73.9 & 8.8 & $1.500(0.783,2.876)$ & 0.238 \\
\hline 2008 & 23 & 6.3 & 87.0 & 7.9 & $1.480(0.772,2.836)$ & 0.058 \\
\hline 2011 & 28 & 7.7 & 89.3 & 10.5 & $1.832(0.979,3.431)$ & 0.087 \\
\hline 2012 & 27 & 7.3 & 85.2 & 9.6 & $1.735(0.923,3.261)$ & 0.080 \\
\hline 2013 & 27 & 7.4 & 81.5 & 9.0 & $1.756(0.934,3.301)$ & 0.116 \\
\hline 2014 & 27 & 7.0 & 96.3 & 8.6 & $1.658(0.882,3.117)$ & 0.122 \\
\hline 2015 & 27 & 6.9 & 88.9 & 9.0 & $1.646(0.875,3.094)$ & 0.006 \\
\hline 2016 & 38 & 9.7 & 86.8 & 13.2 & $2.323(1.278,4.223)$ & 0.165 \\
\hline 2017 & 26 & 6.6 & 84.6 & 9.2 & $1.568(0.831,2.961)$ & 0.008 \\
\hline 2018 & 37 & 9.5 & 81.1 & 14.3 & $2.264(1.243,4.125)$ & \\
\hline
\end{tabular}

Note: Missing data for the year of 2009-2010; the estimated proportion of deaths due to diabetes was $1.01 \%$ ( 317 cases of diabetes vs. 31,262 total cases) in men. \#\# adjusted for age group (0-9, 10-19, 20-29, 30-39, 40-49, 50-59, 60-69, 70-79, 80+). Per-year increment MRR (95\%CI): 1.041 (1.014, 1.069), $\mathrm{p}=0.003$. \$ Crude rate per 100,000 person-years; \$ Age-standardized rate per 100,000 person-years using the World Health Organization standard population for 2000-2025; \# Proportion of death cases aged under 70 year-olds. When combined all cases from 2005-2018 in men, WHO-ASR: 9.4 per 100,000 person-years.

Table 3: Mortality due diabetes in women from 2005 to 2018 in Lang Son province.

\begin{tabular}{|c|c|c|c|c|c|c|}
\hline Year & Case & Crude rate $\mathbf{9}$ & $\mathbf{0 < 7 0 ~ \#}$ & WHO-ASR \$ & MRR (95\%CI) \#\# & p \\
\hline 2005 & 7 & 1.9 & 57.1 & 1.9 & 1 (reference) & 0.642 \\
\hline 2006 & 9 & 2.4 & 55.6 & 2.8 & $1.264(0.471,3.395)$ & 0.373 \\
\hline 2007 & 11 & 3.0 & 72.7 & 3.5 & $1.538(0.596,3.967)$ & 0.525 \\
\hline 2008 & 5 & 1.3 & 40.0 & 1.6 & $0.689(0.219,2.172)$ & 0.824 \\
\hline 2011 & 8 & 2.2 & 50.0 & 2.5 & $1.122(0.407,3.093)$ & 0.852 \\
\hline 2012 & 8 & 2.1 & 25.0 & 2.3 & $1.101(0.399,3.037)$ & 0.834 \\
\hline 2013 & 8 & 2.2 & 37.5 & 2.5 & $1.115(0.404,3.074)$ & 0.252 \\
\hline 2014 & 13 & 3.3 & 69.2 & 3.7 & $1.711(0.683,4.288)$ & 0.104 \\
\hline 2015 & 16 & 4.0 & 50.0 & 4.7 & $2.090(0.860,5.080)$ & 0.744 \\
\hline 2016 & 9 & 2.3 & 44.4 & 2.8 & $1.179(0.439,3.165)$ & 0.293 \\
\hline 2017 & 4 & 1.0 & 0.0 & 1.1 & $0.517(0.151,1.766)$ & 0.449 \\
\hline 2018 & 11 & 2.8 & 27.3 & 3.0 & $1.442(0.559,3.721)$ & 0 \\
\hline
\end{tabular}

Note: Missing data for the year of 2009-2010; the estimated proportion of deaths due to diabetes was $0.61 \%$ (109 cases of diabetes vs. 17,990 total cases) in women. \#\# adjusted for age group $(0-9,10-19,20-29,30-39,40-49,50-59,60-69,70-79,80+)$. Per-year increment MRR (95\%CI): $1.011(0.967,1.057), \mathrm{p}=0.619$. \$ Crude rate per 100,000 person-years; \$ Age-standardized rate per 100,000 person-years using the World Health Organization standard population for 2000-2025; \# Proportion of death cases aged under 70 year-olds. When combined all cases from 2005-2018 in women, WHO-ASR: 2.7 per 100,000 person-years.

\section{Discussion}

We observed a significant increasing trend of DM from 2005 to 2018 in a mountainous province of Lang Son in Viet Nam, especially in men. The risk of developing DM in men was 3.5 times higher than in women. The findings suggested that DM has to be alarming public health issues in this remote and disadvantaged population and people living in this area. The strengthens of the study included a long period of mortality registration for the whole provincial population for all causes including DM to address the time trend and the proportion of premature deaths under the age of 70 years, about three-fourth of total DM deaths. The present findings are basic information for further evident based public health policy plans and the decision to reduce the burden of DM and non-communicable diseases in the country. The mechanism of developing DM is unclear to date. The features of DM included rising glucose levels in the patient's blood because the patient's body cannot create enough of the hormone insulin or cannot effectively use the insulin it creates. Insulin is an essential hormone 
generated in the pancreas. The hormone metabolized glucose from the circulation systems to enter the body's cells where that glucose is converted into energy [1].

An unhealthy diet continues to be a major public health issue worldwide, especially in low-andmiddle-incomecountries, including Viet Nam. The underlying etiology of DM and non-communicable diseases are partly due to unhealthy diet and lifestyles. Red meat intake is a significantly increased risk of DM based on the results of three prospective cohort studies in the U.S. [23]. Meat cooking methods are associated with the risk of DM [24]. The underlying mechanism of red meat intake and meat cooking methods might be the formed chemical dietary carcinogens of heterocyclic amines [24] due to cooking meats at high temperatures during a long time $[25,26]$. Heterocyclic amines included PhIP are generated when meats are cooked at high cooking temperatures resulted from the reaction between amino acids, sugars, and creatinine $[27,28]$. Over twenty types of heterocyclic amines have been identified in cooked meats, and concentrations appear to increase with higher temperatures and longer duration of cooking [29,30]. The other established risk factor of DM is tobacco smoking. Both active and passive tobacco smoking are risk factors for developing DM [3133]. Both red meat intake [34] and tobacco smoking [35] are highly prevalent in Viet Nam that might be responsible for the occurrence of DM with an alarmingly increasing trend, especially in men.

There were a total of 426 diabetic deaths from 2005 to 2018, accounting for $0.86 \%$ of all-causes deaths in Lang Son province. This proportion of mortality due to DM is lower than the WHO report in 2016 on a national scale, with $3 \%$ of deaths due to DM [5]. The diabetes mortality rate also tended to increase over the years, suggesting that there is still a significant limitation in DM's management and treatment in this area. The WHO-ASR mortality rate of DM in Lang Son province was 5.8 deaths per 100,000 people. This is much lower than the United States (U.S.) in 2017, with 25.7 deaths per 100,000 people [36]. Another study in rural America regions also showed a much higher crude mortality rate for the entire population (1999 - 2015), which ranged from 20.5 to 34.2 per 100,000 people [37]. It should be taken into consideration that there are differences in geographic locations as well as the population groups and their lifestyles of these studies, which can lead to disparities in the results. The age-adjusted mortality rate using the WHO standard population was 5.8 deaths per 100,000 people, which was quite trivial compared with 39.34 deaths per 100,000 people in Romania (1998 - 2015) and 23.3 deaths per 100,000 people in Panama (2001 - 2011).

Although there was a significant increase in the age-adjusted mortality rate from 3.5 in 2005 to 8.0 in 2018, remarkable fluctuations between the years over the 12 years were recorded. A similar trend was also witnessed with the mortality rate even after adjusting for both age and gender. This indicates possible differences in the mortality rate in terms of different genders and age groups. However, the mortality rate among diabetic patients in the province still increased generally, with per-year increment MRR was 3.3\% compared to the initial year 2005. This finding was in contrast with the result of a study in Denmark (2004) [38], with a reduction of $4.0 \%$ per year in terms of the mortality rate among people with DM. Another study in Simcoe Muskoka and Ontario provinces of Canada also showed a similar downward trend from 2000 to 2015 [39]. The increasing trend of Lang Son province suggests a lack of early and effective treatment for diabetic patients. Unfortunately, with the collected data, we were unable to analyze and confirm this hypothesis. A limitation of this study is that data available is not represented for the national level and possibly underestimation, because the estimated sensitivity of DM mortality registration is under $60 \%$ in the other provinces in Viet Nam and the data of the Lang Son province will share the same situation. More in-depth studies are needed to accurately evaluate the mortality rate to help policymakers produce appropriate measures to reduce the risk of death from diabetes and to treat and prevent the prevention of this disease is more effective.

\section{Conclusion}

Our study has shown that the mortality rate of diabetic patients has increased continuously over the last decades and a large proportion of premature deaths under the age of 70 years in Lang Son province. The rate increased constantly across all the age groups, and higher mortality was seen in men. These trends suggest continued efforts to improve the treatment of diabetes and its complications are needed. Besides, the absence of a systematic record of mortality rate due to DM at a national scale requires urgent attention and further researches.

\section{Conflicting Interest}

There are no conflicts of interest to disclose.

\section{Acknowledgment}

We are grateful to all staff of the Lang Son's Health facilities involved in data collection, supervision, monitoring, and data management. There were no other conflicts of interest. All authors had input into the final version of the paper.

\section{Author Contribution}

Conceptualization: NTL. Data curation: NVL. Formal analysis: NTL. Funding acquisition: NTL. Methodology: NTL, NVL, LCN. Project administration: NTL, NVL. Visualization: NTH, NTL. Writing - original draft: NTH, NTL, LCN. Writing - review \& editing: NTL, NTH, NBV, LCN. 


\section{References}

1. (2019) IDF (International Diabetes Federation), IDF Diabetes Atlas $\left(9^{\text {th }}\right.$ Edn.)., Brussels, Belgium.

2. JE Shaw, RA Sicree, P Zimmet (2010) Global estimates of the prevalence of diabetes for 2010 and 2030. Diabetes research and clinical practice 87(1): 4-14.

3. (2015) IDF (International Diabetes Federation), IDF Diabetes Atlas $7^{\text {th }}$ Edn.)., Brussels, Belgium.

4. K Ogurtsova, JD da Rocha Fernandes, Y Huang, U Linnenkamp, L Guariguata, et al. (2017) I.D.F.D Atlas, Global estimates for the prevalence of diabetes for 2015 and 2040. Diabetes Res Clin Pract 128: 40-50.

5. (2016) WHO (World Health Organization), Diabetes country profiles, 2016.

6. (2016) WHO (World Health Organization), The growing burden of diabetes in Viet Nam, World Health Organization (WHO).

7. KN Thy (2015) Diabetes in Viet Nam, Annals of global health 81: 870873.

8. Pham Ngoc Minh, Eggleston Karen (2016) Prevalence and determinants of diabetes and prediabetes among Vietnamese adults. Diabetes Research and Clinical Practice 113: 116-124.

9. A Zabetian, IM Sanchez, KMV Narayan, CK Hwang, MK Ali (2014) Global rural diabetes prevalence: a systematic review and meta-analysis covering 1990â€"2012. Diabetes research and clinical practice 104(2): 206-213.

10. (2010) O General Statistics, The 2009 Viet Nam population and housing census: completed results, Statistical Publishing House, Hanoi.

11. (2020) GSO O Vietnam, Socio-economic Statistical Data of 63 provinces and cities, Hanoi.

12. LV Nghia (2020) Status of injury mortality, population-based injury mortality registration in Lang Son province, Viet Nam during 2016-2018. Lang Son Provincial Health Department, Center for Disease Control of Lang Son province, p. 62

13. LV Nghia (2017) Study on work-related mortality in Lang Son province during 2011-2014, Dept. of Occupational Health, Hanoi Medical University, Library of Hanoi Medical University, p. 55.

14. (2018) MOH, Health Statistics Yearbook 2016, Ministry of Health, Ha Noi, Vietnam.

15. LT Ngoan, NT Lua, LT Hang (2007) Cancer mortality pattern in Viet Nam. Asian Pac J Cancer Prev 8(4): 535-538.

16. LT Ngoan (2006) Development of population-based cancer mortality registration in the North of Viet Nam, Asian Pac J Cancer Prev 7(3): 381384.

17. MR Stevenson, DV Hung, HT Huong, LTM Anh, NTH Tu (2015) Evaluation of the Vietnamese A6 Mortality Reporting System: All-Cause Mortality. Asia-Pacific Journal of Public Health 27(7): 733-742.

18. MR Stevenson, LT Ngoan, DV Hung, NTH Tu, AL Mai, et al. (2012) Evaluation of the Vietnamese A6 mortality reporting system: injury as a cause of death3 Inj Prev 18(6): 360-364.

19. M Stevenson, DV Hung, TH Hoang, L Mai Anh, T Nguyen Thi Hong, et al (2015) Evaluation of the Vietnamese A6 Mortality Reporting System: All-Cause Mortality. Asia Pac J Public Health 27(7): 733-742.

20. NP Hoa, C Rao, DG Hoy, ND Hinh, NT Chuc, et al. (2012) Mortality measures from sample-based surveillance: evidence of the epidemiological transition in Viet Nam. Bull World Health Organ 90(10): 764-772.
21. NV Thuong, LQ Minh, M Stevenson, LT Linh, LT Ngoan (2017) Evaluation of a Population-based Intervention to Improve the Vietnamese Mortality Reporting System. Southeast-Asian J of Sciences 5: 194-204.

22. TT Hong, NP Hoa, SM Walker, PS Hill, C Rao (2018) Completeness and reliability of mortality data in Viet Nam: Implications for the national routine health management information system. PLoS One 13(1): e0190755.

23. A Pan, Q Sun, AM Bernstein, JE Manson, WC Willett, et al. (2013) Changes in red meat consumption and subsequent risk of type 2 diabetes mellitus: three cohorts of US men and women. JAMA Intern Med 173(14): 13281335.

24. G Liu, G Zong, K Wu, Y Hu, Y Li, et al. (2018) Meat Cooking Methods and Risk of Type 2 Diabetes: Results From Three Prospective Cohort Studies. Diabetes Care 41(5): 1049-1060.

25. (2014) NTP (National Toxicology Program), Report on Carcinogens, Thirteenth Edition: Heterocyclic Amines (Selected), U.S. Department of Health and Human Services: National Toxicology Program.

26. (1993) IARC (International Agency for Research on Cancer), Some Naturally Occurring Substances: Food Items and Constituents, Heterocyclic Aromatic Amines, and Mycotoxins, in WHO-IARC, IARC Monographs on the Evaluation of Carcinogenic Risks to Humans, WHOIARC, Lyon France, pp. 165-229.

27. JE Hodge (1953) Dehydrated foods, the chemistry of browning reactions in model systems. J Agric Food Chem 1: 928-943.

28. LC Maillard (1912) Action of amino acids on sugars. Formation of melanoidins in a methodical way. Compt Rend 154: 66.

29. RJ Turesky, AK Goodenough, W Ni, L McNaughton, DM LeMaster, et al. (2007) Identification of 2-amino-1,7-dimethylimidazo4,5-g.quinoxaline: an abundant mutagenic heterocyclic aromatic amine formed in cooked beef. Chem Res Toxicol 20(3): 520-530.

30. T Sugimura (1997) Overview of carcinogenic heterocyclic amines. Mutat Res 376(1-2): 211-219.

31. D Liao, L Ma, J Liu, P Fu (2019) Cigarette smoking as a risk factor for diabetic nephropathy: A systematic review and meta-analysis of prospective cohort studies. PLoS One 14: e0210213.

32. S Carlsson, T Andersson, M Araghi, R Galanti, A Lager, et al. (2017) Smokeless tobacco (snus) is associated with an increased risk of type 2 diabetes: results from five pooled cohorts. J Intern Med 281(4): 398-406.

33. X Wei, S Yu (2014) A meta-analysis of passive smoking and risk of developing Type 2 Diabetes Mellitus. Diabetes Res Clin Pract 107(1): 9-14.

34. (2010) Ministry of Health, National Institute of Nutrition, National Nutritional Survey 2009-2010, Medical Publishing House, Hanoi city.

35. HV Minh, KB Giang, NB Ngoc, PT Hai, DT Huyen, et al. (2017) Prevalence of tobacco smoking in Viet Nam: findings from the Global Adult Tobacco Survey 2015. Int J Public Health 62: 121-129.

36. KD Kochanek, J Xu, SL Murphy, AM Minino, HC Kung (2019) Deaths: Final Data for 2017. National Vital Statistics Reports. National Vital Statistics Reports 68(9): 1-77.

37. TH Callaghan, SD Towne, J Bolin, AO Ferdinand (2017) Diabetes mortality in rural America: 1999: 2015, Policy Brief. Southwest Rural Health Research Center, p. 4.

38. B Carstensen, JK Kristensen, P Ottosen, K Borch Johnsen (2008) The Danish National Diabetes Register: trends in incidence, prevalence, and mortality, Diabetologia 51(12): 2187-2196.

39. (2015) Simcoe Muskoka District Health Unit, Diabetes Mellitus (DM) Mortality. 
ISSN: 2574-1241

DOI: $10.26717 /$ BJSTR.2021.39.006374

Ngoan Tran Le. Biomed J Sci \& Tech Res

(C) $(9)$ This work is licensed under Creative

Submission Link: https://biomedres.us/submit-manuscript.php

\begin{tabular}{ll} 
BIOMEDICAL & Assets of Publishing with us \\
RESEARCHES & - Global archiving of articles \\
\hline Immediate, unrestricted online access & - Rigorous Peer Review Process \\
& - Authors Retain Copyrights \\
\end{tabular}

